\title{
Tooth hard tissue stimulates bone remodeling as a potential motive force during tooth eruption
}

\author{
YOUYANG ZHENG ${ }^{1}$, JIALI ZHANG $^{1}$, XIAOLIN CAO $^{2}$, WEILIAN SUN $^{1}$, YANMIN WU $^{1}$ and LILI CHEN ${ }^{1}$ \\ ${ }^{1}$ Department of Stomatology, The Second Affiliated Hospital, School of Medicine, Zhejiang University, \\ Hangzhou, Zhejiang 310009; ${ }^{2}$ Department of Otolaryngolgy Head and Neck Surgery, \\ Hangzhou First People's Hospital, Hangzhou, Zhejiang 310006, P.R. China
}

Received November 10, 2011; Accepted February 14, 2012

DOI: $10.3892 / \mathrm{mmr} .2012 .804$

\begin{abstract}
Tooth eruption is an orientating action which migrates teeth coronally during life, even in bone or after occlusion contact is lost. The eruption pathway is due to bone resorption near the enamel crown and bone deposition around the cementum-covered roots. Due to their proximity to bone resorption and deposition, we hypothesize that the hard tissues enamel, dentine or cementum are important during eruption. In the present study, extracted human teeth were cut into enamel samples, enamel-dentine samples or dentine-cementum samples, and implanted into bone defects in rabbit tibia. Hematoxylin and eosin, tartrate-resistant acid phosphatase activity, tetracycline tracing and scanning electron microscopy were used to investigate bone resorption and deposition 1-8 weeks after surgery. The results showed that resorption lacunae with numerous osteoclasts were observed facing enamel and significant new bone deposition occurred near the cementum surface, compared to other hard tooth surfaces. These findings indicate that the enamel crown may stimulate bone resorption and initiate the eruption pathway, and that the cementum root may stimulate bone deposition. Bone regeneration initiated by tooth hard tissues may be a potential motive force during tooth eruption.
\end{abstract}

\section{Introduction}

The process of tooth eruption can be divided into five stages: pre-eruptive movements, intraosseous eruption, mucosal penetration, pre-occlusal and post-occlusal eruption. Since developing teeth move in three dimensions and increase in size within the alveolar process prior to active eruption, tooth

Correspondence to: Dr Youyang Zheng, Department of Stomatology, The Second Affiliated Hospital, School of Medicine, Zhejiang University, 88 Jiefang Road, Hangzhou, Zhejiang 310009, P.R. China

E-mail: zyyzeyy@163.com

Key words: tooth eruption, bone remodeling, enamel, dentine, cementum eruption exerts an orientating action, and teeth migrate coronally during the second to fourth decades of life. To the best of our knowledge, teeth that are impacted upside down are not likely to allow root eruption, and when occlusional contact is lost, teeth over-erupt coronally (1).

Numerous theories of tooth eruption have been suggested, which involve almost all the tissues in or near an erupting tooth (2-4). Although tooth tissue is hard, it is well accepted that the dental follicle (DF) is required for tooth eruption, while the tooth crown appears to exhibit no involvement (5). When a metal replica is placed in the DF, after removal of the third permanent premolar crown just prior to its eruption, occlusal movement of the metal replica continues, while removal of the DF blocks eruption. Investigating the role of the DF alone, without the enamel portion, is technically difficult as separation of the DF and enamel is not possible without EDTA decalcification, and since immunological rejection may occur with metal replicas, the role of the enamel and crown are ignored in tooth eruption.

Roots begin to form early in eruption. Root elongation and bone deposits around the root force the tooth to migrate coronally. Bone formation is regarded as a potential motive force during tooth eruption (6). When a tooth develops and erupts, the DF remains tightly associated with the enamel, and bone deposits remain near the root; therefore, tooth hard tissues appear to be crucial during eruption. The hard tissues, enamel, dentine and cementum, have distinct physicalchemical characteristics, including biological potential $(7,8)$ and permselectivity (9), as enamel contains anisotropic organized hydroxyapatite and cementum is highly mineralized and rarely cellular. We hypothesized that tooth hard tissues affect alveolar bone remodeling and tooth eruption, and that bone remodeling induced by tooth hard tissue may be a potential motive force for tooth eruption. The objective of this study was to determine the effect of tooth hard tissue on the bone activity, using a rabbit tibia implant model.

\section{Materials and methods}

Sample preparation. Human premolars freshly extracted due to orthodontics were used. After cleaning, these premolars were cut into three different sections: enamel samples (E), enamel-dentine samples (ED) and dentine-cementum samples (DC). Samples were 3-mm long, 1-mm wide and 
$1.5-\mathrm{mm}$ thick, with the $3-\mathrm{mm}$ by $1-\mathrm{mm}$ sections cut vertically to enamel rods or dentine tubules on the surface and dentine side, maintaining the cementum surface intact. Artificial porous hydroxyapatite (HA) bars provided by Professor S.F. Zhao (HABP-C 10*10*20; Biomaterial Engineering Research Center, Sichuan University, Sichuan, China) were cut to the same size as the tooth samples. The samples were sterilized by autoclaving prior to further use.

Experimental study. Left posterior limbs of 26 adult New Zealand white rabbits (13 female, 13 male; average weight $2.44 \mathrm{~kg}$, range, 2.00-3.05) were used. The protocol was approved by the local animal research committee. Surgery was performed under aseptic conditions and sedation by injection of $1 \%$ pentobarbital sodium $(30 \mathrm{mg} / \mathrm{kg}$ ) followed by $2 \%$ Lidocaine. Each animal received 5 implanted bone defects on the anterior plane of the tibia near the upper osteoepiphysis, using dental drills with repeated irrigation of physiological saline solution. The defects were placed $\sim 1 \mathrm{~mm}$ deep, separated by a distance of $\sim 4 \mathrm{~mm}$, parallel to the long axis of the bone, away from the medullary cavity. The samples (E, ED, DC and HA) were placed into the implant beds in the indicated directions (Fig. 1) and secured in position by suturing. One bone defect was left empty as a blank control (BK). Surgical procedures were performed by the same individual (Y.Y. Zheng) and assistants. After surgery, animals received antibiotics and analgesics. Animal weights were measured, and local inflammatory reactions and wound healing were observed by visual and manual examinations over the experimental period.

Histological study. Rabbits were sacrificed at 1, 2, 4, 6 or 8 weeks post-surgery ( $\mathrm{n}=4$ each). Specimens were fixed in $10 \%$ formalin for $24 \mathrm{~h}$, decalcified in $0.5 \mathrm{~mol} / 1$ ethylenediamine tetraacetic acid (EDTA) for $\sim 45$ days until flexible, transparent and easily penetrable by a pin, and then paraffin-embedded. Bone defect and implant sections were cut parallel to the bone surface, stained with hematoxylin and eosin (H\&E) and using tartrate-resistant acid phosphatase activity (TRAP stain), a marker of osteoclasts $(10,11)$.

Tetracycline labeling study. Five rabbits were sacrificed respectively at 1,2, 4, 6 and 8 weeks after surgery. One week prior to euthanasia, the rabbits were injected with $50 \mathrm{mg} / \mathrm{kg}$ $3 \%$ hydrochloric tetracycline (Amresco Inc., Solon, OH, USA). After fixation and dehydration, undecalcified ground sections were prepared and observed under ultraviolet incidental light using a Universal Widefield Microscope, Olympus (V435nm, BH-2; Olympus Corporation, Tokyo, Japan), to determine the amount and source of newly formed bone. Six random images were captured in each section implant site and the percentage fluorescent area was determined using Adobe Photoshop 7.0 (Adobe System Inc., San Jose, CA, USA).

Scanning electron microscopy (SEM) study. One rabbit was sacrificed at 4 weeks post surgery. The samples were then fixed in $2.5 \%$ glutaraldehyde at $4^{\circ} \mathrm{C}$ and prepared for SEM using standard techniques.

Index and standard of investigation. Descriptive histology was used to characterize the pattern of new bone formation around

\begin{tabular}{|c|c|c|c|c|}
\hline d & d & c & 1 & l \\
\hline E & ED & DC & $\mathrm{HA}$ & $\mathrm{BK}$ \\
\hline $\mathbf{e}$ & e & d & $r$ & $\mathbf{r}$ \\
\hline
\end{tabular}

Figure 1. Hard tooth surface implant position in rabbit tibiae. Enamel (e), dentine (d) and cementum (c) surface sides of the enamel (E), enameldentine (ED), and dentine-cementum (DC) tooth implants. The right (r) and left (1) sides of hydroxyapatite (HA) samples were implanted. BK is the no implant control.

the implants and degree of implant resorption. The implant area between the host bone and the $3-\mathrm{mm}$ side of the samples was observed; one sample had two opposite 3-mm sides, thus the focus was on 120 sides. The proportion of implant surface in contact with newly formed bone (bone-to-implant contact) was scored as - (no touch), $+(<1 / 3$ touching), $++(1 / 3$ to $2 / 3$ touching) and $+++(>2 / 3$ touching $)$. The number of TRAPpositive cells was quantified in the implant area, and in the tetracycline labelled specimens, the percentage fluorescent area, representing newly formed bone, was determined.

Statistical analysis. Data were analyzed using SPSS software (version 17.0; SPSS Inc., Chicago, IL, USA). The Wilcoxon rank sum test was used to analyze the data of bone-to-implant. The Student's t-test was used to evaluate the number of TRAPpositive cells and area of fluorescence. The results were considered as statistically significant when $\mathrm{p}<0.05$.

\section{Results}

Local inflammatory reactions and wound healing. One rabbit suffered from an open fracture in the tibia after surgery and was rejected, and another recruited. No chronic inflammatory reaction was observed in any group up to 8 weeks post-surgery. Weight bearing capacity in each animal gradually improved as signs of inflammation subsided (within 10 days). Wound healing was uneventful in all 26 cases and no samples were lost or transposed. Additionally, implants in the 26 surviving animals appeared to achieve clinical immobility.

Histological studies. One week after surgery, sections demonstrated mild inflammatory reactions with moderate fibro-collagenization. At the enamel surface sides (e) of E and ED (E-e, ED-e), there were multiple obvious bone resorption fossa, with the presence of TRAP-positive multinucleate cells (Fig. 2A and B). At the E-d, ED-d and the two sides of DC samples, the host cortex demonstrated a lamellar appearance of newly formed bone (Fig. 2C). No bone resorption or bone formation was observed in the gap between the HA implants and the host, where numerous macrophages were detected. In the blank implant site (BK), a small amount of bony tissue formed at the host bone surface.

At weeks 2 and 4, lamellar bone formed bone resorption fossa along with a few small dentine resorption fossa, in the E-e and ED-e implants. A network of woven bone formed and appeared mildly thickened in the E-d, ED-d and DC implants (Fig. 2D-F). HA implants were enveloped by connective tissue with little lamellar bone formation. In the BK control, the bone defect was filled with loosely woven bone. 

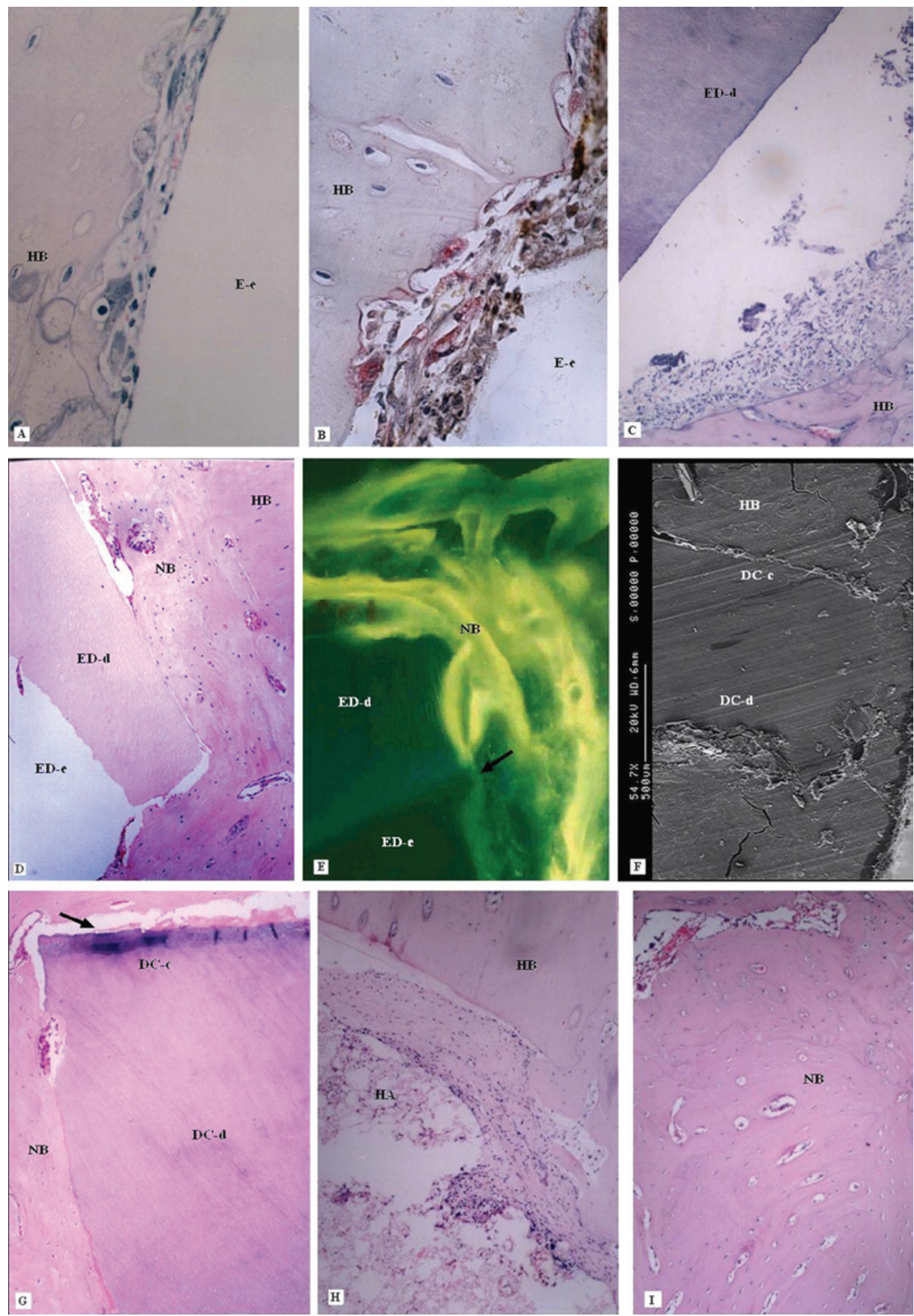

Figure 2. Bone remodeling in different hard tooth surfaces implanted in rabbit tibia showing new bone (NB) and host bone (HB). (A) Osteoclasts at the enamel side of the enamel implant (E-e) 1 week post-surgery; H\&E; magnification, x400. (B) Osteoclasts at E-e 1 week post-surgery; TRAP; magnification, x400), (C) New bone formation at the dentine side of enamel-dentine implant (ED-d) 1 week post-surgery; H\&E; magnification, x40. (D) New bone deposits tightly opposed to ED-d 4 weeks post-surgery; H\&E, magnification, x40. (E) Tetracycline labeling study in enamel-dentine implant 4 weeks post-surgery, arrow indicates the enamel-dentine junction; magnification, x100. (F) SEM indicating tighter contact between host bone and cementum side than the dentine side of dentine-cementum (DC) implants 6 weeks post-surgery. (G) DC implant with fine osseointegration 8 weeks post-surgery, space indicated by arrow is a preparation artifact; H\&E; magnification, x100. (H) Scarcely any new host bone in hydroxyapatite (HA) implant 8 weeks post-surgery; H\&E; magnification, x100. (I) Lamellar bone in no implant defect (BK) 8 weeks post-surgery; H\&E; magnification, x40.

By weeks 6 and 8 , the results were comparable to 2 or 4 weeks, but more advanced (Fig. 2G-I). The BK control was filled with loosely woven bone with larger medullary spaces, compared to cementum surface sides of the DC implant.
In total, 60 tooth samples ( 3 types) and 120 sides (6 types) were observed using H\&E staining. New bone formation was significantly different in the 6 types of sides of the three different implant samples, with DC-c $>$ E-d $>$ ED-d $>$ DC-d $>$ ED-e $>$ E-e. 
Table I. Proportion of bone-to-implant contact in different hard tooth surfaces implanted in rabbit tibia at 1-8 weeks was scored using H\&E staining.

\begin{tabular}{|c|c|c|c|c|c|c|c|c|c|c|c|}
\hline & \multicolumn{2}{|c|}{ Enamel } & \multicolumn{2}{|c|}{ Enamel-dentine } & \multicolumn{2}{|c|}{ Dentine-cementum } & \multicolumn{2}{|c|}{ HA } & \multicolumn{2}{|c|}{ BK control } & \multirow[t]{2}{*}{ Total } \\
\hline & $\mathrm{e}$ & $\mathrm{d}$ & e & $\mathrm{d}$ & $\mathrm{d}$ & $\mathrm{c}$ & $\mathrm{r}$ & 1 & $\mathrm{r}$ & 1 & \\
\hline- & 8 & 1 & 8 & 3 & 6 & 3 & 7 & 8 & 5 & 4 & 53 \\
\hline+ & 7 & 7 & 4 & 5 & 6 & 5 & 6 & 6 & 5 & 6 & 57 \\
\hline++ & 4 & 9 & 6 & 7 & 7 & 5 & 6 & 5 & 6 & 7 & 62 \\
\hline+++ & 1 & 3 & 2 & 5 & 1 & 7 & 1 & 1 & 4 & 3 & 28 \\
\hline Total & 20 & 20 & 20 & 20 & 20 & 20 & 20 & 20 & 20 & 20 & 200 \\
\hline
\end{tabular}

e, enamel side; d, dentine side; c, cementum side; $r$, right; 1 , left. Total tooth implants $(n=60) H=13.8722>x_{0.05(5)}^{2}, p<0.05$. Between Enamel e side and Enamel d side: $\mathrm{D}=26.475>\mathrm{D}_{0.05(114)}, \mathrm{p}<0.05$. Between the Enamel e side and Enamel-dentine $d$ side: $\mathrm{D}=26.175>\mathrm{D}_{0.05(114)}, \mathrm{p}<0.05$. Between the Enamel e side and Dentine-cementum d side: $\mathrm{D}=29.025>\mathrm{D}_{0.01(114)}, \mathrm{p}<0.01$. Between the Dentine-cementum d side and Dentinecementum c side: $\mathrm{D}=20.475>\mathrm{D}_{0.05(114)}, \mathrm{p}<0.05$. For other combinations of sides, $\mathrm{p}>0.05$.

Table II. Area fluorescently labeled with tetracycline in different hard tooth surfaces implanted in rabbit tibiae (\%).

\begin{tabular}{|c|c|c|c|c|c|c|}
\hline \multirow[t]{2}{*}{ Week } & \multicolumn{2}{|c|}{ Enamel $(\mathrm{n}=1)$} & \multicolumn{2}{|c|}{ Enamel-dentine $(\mathrm{n}=1)$} & \multicolumn{2}{|c|}{ Dentine-cementum $(n=1)$} \\
\hline & e $(\%)$ & $\mathrm{d}(\%)$ & e $(\%)$ & $\mathrm{d}(\%)$ & $\mathrm{d}(\%)$ & $\mathrm{c}(\%)$ \\
\hline 1 & 0.87 & 18.56 & 0.33 & 21.36 & 29.45 & 36.19 \\
\hline 2 & 28.17 & 53.15 & 30.05 & 52.88 & 51.32 & 66.48 \\
\hline 4 & 55.32 & 78.67 & 52.98 & 76.34 & 53.95 & 80.07 \\
\hline 6 & 89.47 & 87.62 & 86.69 & 66.99 & 70.72 & 96.41 \\
\hline 8 & 80.23 & 94.12 & 85.54 & 67.82 & 78.16 & 91.00 \\
\hline
\end{tabular}

e, enamel side; $d$, dentine side; c, cementum side.

The bone formation in E-e and DC-c $(p<0.01)$, E-e and ED-d $(\mathrm{p}<0.05)$, E-e and E-d $(\mathrm{p}<0.05)$, DC-d and DC-c $(\mathrm{p}<0.05)$ were significantly different (Table I). At week 2, the differences between the opposite sides of the same tooth sample were the most significant $(\mathrm{p}<0.05)$. However, the differences in bone formation were found not to be significant following a certain period of time. Significantly more new bone formed at the dentine and cementum sides as compared to that of the HA and BK controls $(\mathrm{p}<0.05)$ at the same time. No significant differences were observed between HA or BK $(\mathrm{p}<0.05)$.

The number of osteoclasts, indicated by TRAP-positive multinucleated cells, was higher at the E-e, ED-e, DC-d implant sides, compared to the opposite sides of the same sample, i.e., E-d, ED-d, DC-c (significant at p<0.01, t=5.149; Student's t-test). The total number of osteoclasts in the tooth samples was significantly different between the opposite sides for all weeks, with the exception of week $8(p>0.05)$. No significant differences were observed between HA or BK ( $p>0.05)$.

Fluorescent tetracycline labeling study. Sections revealed a golden yellow fluorescence representing new bone formation in sharp edges, reticulated or lamellar-shaped patterns in the area between the implant and tibia. Thin bands of fluorescent regions surrounded or invaded the HA samples, and the BK control space was filled with loose reticulated golden yellow fluorescence. The fluorescent area percentage was larger at the E-d, ED-d, DC-c sides than the opposite side at E-e, ED-e, DC-d ( $<<0.05$, Student's t-test; Table II). The trend disappeared in the ED sample after 4 weeks.

\section{Discussion}

Effect of different tooth sections on bone remodeling. In our rabbit tibia model, healing was uneventful in all animals and there was little evidence of implant rejection. Teeth have a low composition of organics, but carry transplantation antigens. However, the teeth failed to stimulate immunologic memory in weakly disparate mice (12). Allografts and xenografts of canine teeth showed no significant differences in root resorption (13). In our histological and fluorescent tetracycline labeling study, we found that more new bone was deposited on the cementum side compared to the dentine side, which in turn had more bone than the enamel side of the tooth samples. Moreover, bone densities on the cementum and dentine sides were greater than in the hydroxyapatite or blank control implant beds. More bone resorption and osteoclasts were detected around the enamel side than the dentine or cementum sides of implants. These results suggest that dental roots induce new bone formation and are capable of accelerating bone healing, while the enamel or dental crown stimulates bone resorption. 
These trends were most obvious in the early periods, 1-2 weeks after surgery, and gradually disappeared as the bone healed, in accordance with the regularity of bone remodeling, the thin thickness of the tooth section implants and short model time-frame.

Tooth eruption and tooth hard tissues. During the process of tooth eruption, teeth are known to migrate coronally during their life, even within bone or after occlusion contact is lost. Bone resorption near the enamel crown and bone deposition around the cementum-covered roots occur during eruption. Our study has shown that the eruption pathway may be initiated by special properties of enamel, which contribute to bone resorption, and cementum-covered roots, which contribute to bone formation. As teeth do not touch alveolar bone, it is probably the DF connective tissue around the tooth that transduces the effects of bone resorption or formation to cause eruption. Most likely, these metabolic events are initiated by enamel, and later sustained and coordinated by the dental follicle via local signals involving the follicle or adjacent tissue proteins and growth factors.

The mucosal penetration and pre-occlusal eruption phases are rapid, and mostly due to bone formation and root elongation, which slow when the occlusal plane is approached. We propose that the effects of bone formation are balanced by occlusal tension, which then evokes bone resorption via mechanotransduction. Bone deposits near the root or cementum compensate for occlusal attrition. If occlusal contact is lost and mechanotransduction disappears, tooth eruption rate is likely to increase, leading to over-eruption.

The results of this study suggest that the dental root is important in alveolar bone formation. This hypothesis may explain reduced atrophy in alveolar bone containing residual roots without occlusion, as it is generally accepted that alveolar bone atrophy in the area of anodontia is due to an absence of mechanical occlusion force. The phenomenon of bone atrophy in the area of anodontia or hypodontia may be due to the absence of stimulation by tooth hard tissue.

In conclusion, this study suggests that tooth hard tissue directly affects bone remodeling and tooth occlusal eruption: enamel stimulates bone resorption and cementum-covered roots stimulate bone formation. Over-eruption occurs when the balance between bone deposits, stimulated by dental root and bone resorption due to mechanical tension is broken.

Further experiments are required to explore the role of other components, such as the DF and periodontal ligament in tooth. However, this study has shown that tooth hard tissue is involved in tooth eruption.

\section{Acknowledgements}

The authors thank Professors S.F. Zhao and J.A. Hu, Stomatology Faculty of Zhejiang University, Medical School, for their advice on this project and discussions on the manuscript, as well as for providing the facilities used in this study. The study was supported by a grant from the Bureau of Education, Zhejiang Province, China (Y200804437).

\section{References}

1. Xu J, Jiang Y and Dong Q: Experimental study on supraeruption of the unopposed miniature pig molars. Zhonghua Kou Qiang Yi Xue Za Zhi 32: 276-278, 1997.

2. Marks SC Jr and Schroeder HE: Tooth Eruption: theories and Facts. Anat Rec 245: 374-393, 1996.

3. Wise GE, Frazier-Bowers S and D'Souza RN: Cellular, molecular, and genetic determinants of tooth eruption. Crit Rev Oral Biol Med 13: 323-334, 2002.

4. Wise GE and King GJ: Mechanisms of tooth eruption and orthodontic tooth movement. J Dent Res 87: 414-434, 2008.

5. Marks SC Jr and Cahill DR: Experimental study in the dog of the non-active role of the tooth in the eruptive process. Arch Oral Biol 29: 311-322, 1984.

6. Wise GE, Yao SM and Henk WG: Bone formation as a potential motive force of tooth eruption in the rat molar. Clin Anat 20: 632-639, 2007.

7. Qiao YN and Zhang JL: Biological potential and ion transferred study on tooth. Chem J Chinese Universities 16: 1462-1463, 1995.

8. Zhang JL, Zheng YY and Su JM: The measurement and research of surface potentials of human tooth in vitro. The Proceedings of the 23rd Annual International Conference of the IEEE Engineering in Medicine and Biology Society, 2001.

9. Vogel GL, Mao Y, Carey CM and Chow LC: Changes in the permselectivity of human teeth during caries attack. J Dent Res 76: 673-681, 1997.

10. Sakakura Y, Yajima T and Tsueuga: Confocal laser scanning and microscopic study of tartrate-resistant phosphatese-positive cells in the dental follicle during early morphogenesis of mouse embryonic molar teeth. Arch Oral Biol 43: 353-360, 1998.

11. Yu MX, Jin WF and Wang HF: Observation of the survival period osteoclasts cultivated in vitro with tartrate resistant acid phosphatase (TRAP) staining. J Tanshan Med Col 17: 91-93, 1996.

12. Moore LJ: Tooth allografts fail to stimulate immunologic memory in mice. J Dent Res 58: 451-460, 1979.

13. Yang XM and Ni HM: Observation of cytoimmunity index on rejection of canine teeth transplantation. J Shanghai Second Med Univ 16: 331, 1996. 